\title{
A CLASS OF PARTIALLY ORDERED LINEAR ALGEBRAS
}

\author{
RALPH DEMARR
}

ABstract. We consider a special type of partially ordered linear algebra which is like an algebra of real-valued functions. We show that various natural properties characterize this type of algebra. These natural properties relate the algebraic and order structures to each other.

A pola (denoted by $A$ ) is a real linear associative algebra which is partially ordered so that it is a directed partially ordered linear space and $0 \leqq x y$ whenever $x, y \in A, 0 \leqq x, 0 \leqq y$. We also assume that $A$ has a multiplicative identity $1 \geqq 0$. A Dedekind $\sigma$-complete pola (dsc-pola) $A$ is one having the property: if $x_{n} \in A, 0 \leqq \cdots \leqq x_{2} \leqq x_{1}$, then $\inf \left\{x_{n}\right\}$ exists. Order convergence is defined as usual. A dsc-pola $A$ has the Archimedean property: if $x, y \in A$ and $n x \leqq y$ for every positive integer $n$, then $x \leqq 0$. For more details and examples see the references.

The simple example of interest to us here is the dsc-pola $A$ of all realvalued functions defined on some nonempty set, where the algebraic operations and the partial order are defined pointwise. We note that $A$ has the following property:

$\mathrm{P}_{1}$ : If $x \in A$ and $x \geqq 1$, then $x$ has an inverse and $x^{-1} \geqq 0$.

If we now consider an arbitrary dsc-pola $A$ which has property $\mathrm{P}_{1}$, then we can show that $A$ is much like an algebra of real-valued functions; however, the operations may only be defined "almost everywhere" (see example 5 of [4]). Some of the basic properties are given in the following theorem.

THEOREM 1. If $A$ is a dsc-pola which has property $\mathrm{P}_{1}$, then multiplication of elements in $A$ is commutative and $A$ is a lattice. Furthermore, $x^{2} \geqq 0$ for all $x \in A$ and if $y \in A$ and $y \geqq 0$, then there exists $a$ unique $z \in A$ such that $z^{2}=y$ and $z \geqq 0$.

This theorem was proved by the author but it appears as the necessary introduction to the thesis of his former student, T. Dai, who showed in

Received by the editors September 18, 1972.

AMS (MOS) subject classifications (1970). Primary 06A70; Secondary 15A45.

Key words and phrases. Dedekind $\sigma$-complete partially ordered linear algebra, algebra of real-valued functions, matrix inequalities, $f$-ring.

(c) American Mathematical Society 1973 
addition that $A$ (having property $\mathrm{P}_{\mathbf{1}}$ ) is an $f$-ring $[1, \mathrm{p} .403]$. The reader is referred to Dai's paper [2] for examples and proofs. The purpose of this paper is to show that various natural properties for a dsc-pola $A$ imply that $A$ has property $\mathrm{P}_{1}$.

LEMMA 1. Let $A$ be a dsc-pola. If $x \leqq 1$ and there exists $y \geqq 0$ such that $1 \leqq x y$ or $1 \leqq y x$, then $x$ has an inverse and $x^{-1} \geqq 1$. From this it follows that if $1 \leqq u \leqq v$ and $v$ has an inverse and $v^{-1} \geqq 0$, then $u$ has an inverse and $u^{-1} \geqq 0$.

For the proof see Proposition 3 and its corollary in [3].

Lemma 2. Let $A$ be a dsc-pola which has the property: if $x \in A$ and $x \geqq 0$, then there exists a sequence $\left\{x_{n}\right\}$ of elements from $A$ such that $0 \leqq$ $x_{n} \leqq n 1$ for all $n$ and $o$-lim $x_{n}=x$. Then $A$ has property $\mathbf{P}_{1}$.

For the proof see (v) of Lemma 1.6.6 in [2].

The following two properties concern one-sided factoring of one element by another.

THEOREM 2. Let $A$ be a dsc-pola which has the property: if $y_{1}, y_{2} \in A$ and $0 \leqq y_{1} \leqq y_{2}$, then there exists $w \in A$ such that $w \geqq 0$ and $y_{1}=w y_{2}$. Then $A$ has property $\mathbf{P}_{1}$.

Proof. Take any $x \in A$ such that $x \geqq 1 \geqq 0$. Hence, there exists $w \in A$ such that $w \geqq 0$ and $w x=1$. Since $w \geqq 0$ and $x \geqq 1$, we have $w \leqq 1$. Using Lemma 1 , we see that $x^{-1}=w \geqq 0$.

THeOREM 3. Let $A$ be a dsc-pola which has the property: if $y_{1}, y_{2} \in A$ and $1 \leqq y_{1} \leqq y_{2}$, then there exists $w \in A$ such that $w \geqq 1$ and $w y_{1}=y_{2}$. Then $A$ has property $\mathrm{P}_{1}$.

Proof. Take any $x \in A$ such that $x \geqq 1$. Since $1 \leqq x \leqq x+1$, there exists $w \in A$ such that $w \geqq 1$ and $w x=x+1$. Hence, $(w-1) x=1$, where $w-$ $1 \geqq 0$. Thus, $w-1 \leqq 1$ and we may again use Lemma 1 to show that $x^{-1}=$ $w-1 \geqq 0$.

The following is a decomposition property for multiplication in a dscpola which is commutative.

THEOREM 4. Let A be a commutative dsc-pola which has the property: if $y \in A, y \geqq 0$ and $0 \leqq w \leqq y^{2}$, then there exist elements $u, v \in A$ such that $0 \leqq u \leqq y, 0 \leqq v \leqq y$ and $u v=w$. Then $A$ has property $\mathbf{P}_{\mathbf{1}}$.

Proof. Take any $x, y \in A$ such that $1 \leqq x \leqq x+1 \leqq y$. Thus, $0 \leqq y^{2}-$ $1 \leqq y^{2}$. Hence, we may find $u, v \in A$ such that $0 \leqq u \leqq y, 0 \leqq v \leqq y$, and $u v=$ $y^{2}-1$. We see easily that $1=y(y-u)+u(y-v)$. We remark that this is the only place we use commutativity. Using the inequalities given above, one can easily show that $0 \leqq y-u \leqq 1, u \geqq 1$ and then $0 \leqq y-v \leqq 1$. Since 
$1+(y-u)(y-v)=y(2 y-u-v)$ and $0 \leqq(y-u)(y-v) \leqq 1$, we see that $\left(\frac{1}{2}\right) y(2 y-u-v) \leqq 1$ and then by using Lemma 1 , we obtain $[y(2 y-u-v)]^{-1} \geqq$ 0 . By using Lemma 1 twice, one can show first that $y^{-1} \geqq 0$ and then $x^{-1} \geqq 0$.

At the end of the paper a counterexample will be given to show that $A$ must be commutative in the previous theorem. However, we can drop commutativity if we use a stronger decomposition property as follows.

TheORem 5. Let $A$ be a dsc-pola which has the property: if $y \in A$, $y \geqq 0$ and $0 \leqq w \leqq y^{2}$, then there exists $u \in A$ such that $0 \leqq u \leqq y$ and $u^{2}=w$. Then $A$ has property $\mathrm{P}_{1}$.

Proof. Take any $x, y \in A$ such that $1 \leqq x \leqq x+1 \leqq y$. Thus, $0 \leqq y^{2}-$ $1 \leqq y^{2}$. Hence, we may find $u \in A$ such that $0 \leqq u \leqq y$ and $u^{2}=y^{2}-1$. We see easily that $1=y(y-u)+(y-u) u$. Using the inequalities given above, one can easily show that $0 \leqq y-u \leqq 1$. Since $0 \leqq y-u$, we see that $y(y-u) \leqq$ 1 and $y(y-u)(1+u)=y(y-u)+y(y-u) u \geqq 1$. Using Lemma 1 twice, we can first show that $[y(y-u)]^{-1} \geqq 0$ and then $y^{-1} \geqq 0$. Using Lemma 1 again, we can show that $x^{-1} \geqq 0$.

Next we consider an order-reversing property for left inverses.

THEOREM 6. Let $A$ be a dsc-pola which has the property: if $y_{1}, y_{2} \in A$ and $1 \leqq y_{1} \leqq y_{2}$, then there exist $w_{1}, w_{2} \in A$ such that $w_{2} \leqq w_{1}$ and $w_{1} y_{1}=$ $w_{2} y_{2}=1$. Then $A$ has property $\mathbf{P}_{1}$.

Proof. Take any $x \in A$ such that $1 \leqq x \leqq x+1$. There exist $w_{1}, w_{2} \in A$ such that $w_{2} \leqq w_{1}$ and $w_{1} x=w_{2}(x+1)=1$. Hence, $\left(w_{1}-w_{2}\right) x=w_{2}$ and $\left(w_{1}-w_{2}\right) x(x+1)=1$. Since $x(x+1) \geqq 1$ and $0 \leqq w_{1}-w_{2}$, we have $w_{1}-w_{2} \leqq 1$. Using Lemma 1 twice, we can first show that $[x(x+1)]^{-1}=w_{1}-w_{2} \geqq 0$ and then $x^{-1} \geqq 0$.

The next property concerns generalized inverses.

THEOREM 7. Let $A$ be a dsc-pola which has the property: if $z \in A$ and $z \geqq 1$, then there exists $w \in A$ such that $w \geqq 0$ and $z w z=z$. Then $A$ has property $\mathrm{P}_{1}$.

Proof. From the above it follows that if $v \in A, v \geqq 1$ and $v$ has an inverse, then $v^{-1} \geqq 0$. Let us now take $w$ and $z$ as in the statement of the theorem. If we put $u=w z$, then $0 \leqq u=u^{2}$. Since $1+n u \geqq 1$ and $1+n u$ has an inverse for every positive integer $n$, we obtain $0 \leqq(1+n u)^{-1}=1-$ $(n / n+1) u$ for all $n$. Using the Archimedean property, we obtain $w z=u \leqq 1$. Since $z w z=z \geqq 1$, we obtain $(w z)^{-1} \geqq 1$ by using Lemma 1. By again using Lemma 1 we obtain $z^{-1} \geqq 0$.

The following question is unanswered. 
Question. Let $A$ be a dsc-pola which has the property: if $x \in A$, then $x^{2} \geqq 0$. Does $A$ have property $\mathrm{P}_{1}$ ?

We can answer this question in certain special cases.

THEOREM 8. Let $A$ be a dsc-pola which has the properties: $A$ is a lattice and if $x \in A$, then $x^{2} \geqq 0$. Then $A$ has property $\mathrm{P}_{1}$.

PRoof. Take any $z \in A$ such that $z \geqq 0$. Since $(2 n 1-z)^{2} \geqq 0$, we get $0 \leqq z \leqq n 1+(1 / 4 n) z^{2}$ for every positive integer $n$. Since $A$ is a lattice, we may write $z=z_{n}+w_{n}$, where $0 \leqq z_{n} \leqq n 1$ and $0 \leqq w_{n} \leqq(1 / 4 n) z^{2}$ for all $n$. Thus, $o$ $\lim z_{n}=z$. Using Lemma 2 , we see that $A$ has property $\mathbf{P}_{1}$.

Some definitions are necessary for the next two theorems. An element $u \in A$ is called an order unit if $u \geqq 0$ and if for any $x \in A$ there exists a real number $\alpha$ such that $-\alpha u \leqq x \leqq \alpha u$. A dsc-pola $A$ is said to have the PerronFrobenius (PF) property if for every $x \in A, x \geqq 0$, there exists a real number $\lambda>0$ such that $\lambda 1-x$ has an inverse and $(\lambda 1-x)^{-1} \geqq 0$. The name of this property is justified in [4]. A dsc-pola $A$ is said to have the large inverse property if for any $x \in A$ there exists $y \in A$ such that $x \leqq y$ and $y$ has an inverse. The large inverse property plays a key role in Theorem 10 . The next theorem gives a chain of implications showing that the large inverse property is a consequence of other natural properties.

TheOREM 9. Let $A$ be a dsc-pola. If $A$ is finite-dimensional, then $A$ has an order unit. If $A$ has an order unit, then $A$ has the PF property. If $A$ has the PF property, then $A$ has the large inverse property.

Proof. The first implication is a consequence of two facts: $A$ is finite dimensional and $A$ is directed. The proof of the second implication can be found in Theorem 6 of [4]. The proof of the third implication can be found in Proposition 3 of [3].

THEOREM 10. Let $A$ be a dsc-pola which has the large inverse property and also has the property: if $z \in A$, then $z^{2} \geqq 0$. Then $A$ has property $\mathbf{P}_{1}$.

Proof. Take any $x \in A$ such that $x \geqq 1$. Since $A$ has the large inverse property, there exists $y \in A$ such that $x \leqq y$ and $y$ has an inverse. From the second property we obtain $0 \leqq\left(y^{-1}\right)^{2}=\left(y^{2}\right)^{-1}$. Since $1 \leqq x \leqq y \leqq y^{2}$, we can use Lemma 1 to show that $x^{-1} \geqq 0$.

The final two theorems concern special assumptions about the way an element can be expressed as the difference of two nonnegative elements.

TheOREM 11. Let $A$ be a dsc-pola which has the property: if $x \in A$, then there exists $a \in A$ such that $0 \leqq a \leqq 1, a x \geqq 0$ and $(1-a) x \leqq 0$. Then $A$ has property $\mathbf{P}_{\mathbf{1}}$. 
Proof. Take any $z \in A$ such that $z \geqq 0$. Next select $a_{n} \in A$ such that $0 \leqq a_{n} \leqq 1, a_{n}(n 1-z) \geqq 0$ and $\left(1-a_{n}\right)(n 1-z) \leqq 0$ for every positive integer $n$. Hence, $0 \leqq a_{n} z \leqq n a_{n} \leqq n 1$ and $0 \leqq n\left(1-a_{n}\right) \leqq\left(1-a_{n}\right) z$. From the latter inequalities and the fact that $0 \leqq 1-a_{n} \leqq 1$, we obtain $0 \leqq n\left(1-a_{n}\right) \leqq z$ and then $0 \leqq z-a_{n} z \leqq(1 / n) z^{2}$. Putting $z_{n}=a_{n} z$, we see that $0 \leqq z_{n} \leqq n 1$ and $o$ $\lim z_{n}=z$. We may now use Lemma 2 to show that $A$ has property $\mathrm{P}_{1}$.

The last theorem was proved by the author but credit is due Ralph Gellar. He proved a slightly weaker theorem which inspired the author to work on the following theorem.

THEOREM 12. Let $A$ be a dsc-pola which has the property: if $x \in A$, then there exist $y, z \in A$ such that $y \geqq 0, z \geqq 0, y z=0$ and $x=y-z$. Then $A$ has property $\mathrm{P}_{1}$. (Gellar also assumed that $z y=0$.)

Proof. The key idea involved is that if $a \in A$ and $0 \leqq a^{2} \leqq a$, then $a \leqq 1$. We first prove this fact.

There exist $b, c \in A$ such that $b \geqq 0, c \geqq 0, b c=0$ and $1-a=b-c$. Since $a^{2} \leqq a$, we have $0 \leqq a-a^{2}=a(1-a)=a(b-c)$, which means that $0 \leqq a c \leqq a b$. Hence, $0 \leqq a c^{2} \leqq a b c=0$, which means that $a c^{2}=0$. Since $1 \leqq 1+c=a+b$, we obtain $0 \leqq c^{2} \leqq a c^{2}=0$, which means that $c^{2}=0$. Now there exist $d$, $e \in A$ such that $d \geqq 0, e \geqq 0, d e=0$ and $1-c=d-e$. Therefore, $1 \leqq 1+e=$ $c+d$ so that $e \leqq c e$. Hence, $0 \leqq e \leqq c e \leqq c^{2} e=0$, which means that $e=0$. It follows that $0 \leqq 1-c$ so that $0 \leqq(1-c)^{n}=1-n c$ for every positive integer $n$. From the Archimedean property it follows that $c=0$, which means that $a \leqq 1$.

Now take any $h \in A$ such that $h \geqq 0$. For each positive integer $n$ there exist $y_{n}, z_{n} \in A$ such that $y_{n} \geqq 0, z_{n} \geqq 0, y_{n} z_{n}=0$ and $n 1-h=y_{n}-z_{n}$. Hence, $0 \leqq y_{n}^{2} \leqq y_{n}\left(y_{n}+h\right)=y_{n}\left(n 1+z_{n}\right)=n y_{n}$ for all $n$. Thus, $0 \leqq(1 / n)^{2} y_{n}^{2} \leqq(1 / n) y_{n}$ so that $0 \leqq y_{n} \leqq n 1$ for all $n$. The last inequality is obtained from the result of the preceding paragraph. Since $y_{n} \leqq n 1$, we obtain $z_{n} \leqq h$ for all $n$. Now $n z_{n} \leqq\left(n 1+z_{n}\right) z_{n}=\left(y_{n}+h\right) z_{n}=h z_{n} \leqq h^{2}$ for all $n$, which means that if we define $h_{n}=n 1-y_{n}$, then $0 \leqq h-h_{n} \leqq(1 / n) h^{2}$. Thus, $0 \leqq h_{n} \leqq n 1$ and $o-\lim h_{n}=$ $h$. Using Lemma 2 , we see that $A$ has property $\mathrm{P}_{1}$.

COUNTEREXAMPLES. Let $M$ be the real linear algebra of all 2-by-2 matrices in upper triangular form, where all entries are real. If $M$ is partially ordered entry by entry, then $M$ is a dsc-pola which is not commutative. The reader is invited to use $M$ to verify that the order conditions are necessary in Theorems 2, 3, 5, 6 and 7. For example, look at the proof of Theorem 2. If we take any $x \in M$ such that $x \geqq 1$, then there exists $w \in M$ such that $w x=1$, but it may happen that $w$ not $\geqq 0$. The dsc-pola $M$ has the property described in Theorem 4 but it is not commutative. Note that $M$ is a lattice and has the large inverse property but it does not have 
the other property needed in Theorems 8 and 10 . Also $M$ does not have the properties described in Theorems 11 and 12 .

\section{REFERENCES}

1. G. Birkhoff, Lattice theory, Amer. Math. Soc. Colloq. Publ., vol. 25, Amer. Math. Soc., Providence, R.I., 1967. MR 37 \#2638.

2. T. Dai, On a special class of partially ordered linear algebras, J. Math. Anal. Appl. 40 (1972), 649-682.

3. R. E. DeMarr, On partially ordering operator algebras, Canad. J. Math. 19 (1967), 636-643.

4. —_, A generalization of the Perron-Frobenius theorem, Duke Math. J. 37 (1970), 113-120.

Department of Mathematics, University of New Mexico, Albuquerque, New MeXico 87106 